\title{
Hydrodynamics of Quartz-Crystal-Microbalance DNA Sensors Based on Liposome Amplifiers
}

\author{
Adolfo Vázquez-Quesada $\odot,{ }_{1}^{1}$ Marc Meléndez Schofield $\odot,{ }^{1}$ Achilleas Tsortos $\odot,{ }^{2}$ Pablo Mateos-Gil, ${ }^{2}$ \\ Dimitra Milioni, ${ }^{2}$ Electra Gizeli®, ${ }^{2,3}$ and Rafael Delgado-Buscalioni ${ }^{1, *}$ \\ ${ }^{1}$ Departmento de Fisica de la Materia Condensada, Universidad Autonoma de Madrid, and Institute for \\ Condensed Matter Physics, IFIMAC, Campus de Cantoblanco, Madrid 28049, Spain \\ ${ }^{2}$ Institute of Molecular Biology and Biotechnology, Foundation for Research and Technology-Hellas, Heraklion, \\ Crete 70013, Greece \\ ${ }^{3}$ Department of Biology, University of Crete, Heraklion 71110, Greece
}

(Received 25 February 2020; revised manuscript received 27 May 2020; accepted 29 May 2020; published 24 June 2020)

The quartz-crystal microbalance (QCM) has become an important technique for the detection of biomolecules and their interactions. Acoustic sensing of DNA requires hybridizing the oligonucleotide chain with a nanoparticle that acts as an acoustic amplifier. In this device, the amplifier remains suspended in the liquid, nanometers away from the surface. The hydrodynamics of nonadsorbed nanoparticles in QCM sensing devices has, however, been overlooked in the literature. Here, we show that the acoustic response (shifts in frequency, $\Delta f$, and dissipation, $\Delta D$ ) of the amplifier-target complex is determined by the hydrodynamic wall stress. Our study focuses on an alternative type of DNA sensor based on liposome-DNA complexes and provides a theoretical analysis backed up by experiments with 1-palmitoyl2-oleoyl-sn-glycero-3-phosphocholine liposomes. The target DNA strands are attached on one side to surface-adsorbed neutravidin through a biotin linker, while the other side is anchored to a liposome acting as an amplifier. A range of liposome radii and double-stranded-DNA contour lengths are studied, covering sizes up to the wave penetration depth $(\delta \sim 100 \mathrm{~nm})$. Simulations, based on the immersed boundary method and an elastic-network model, are in excellent agreement with the experiments. We derive an analytical expression for the acoustic impedance, which reflects strong signal amplification as the liposome gets closer to the wall. This enhancement is due to hydrodynamic reflection: the ambient flow induces a stress on the liposome surface, which propagates back to the resonator. For this reason, acoustic signals are extremely sensitive to the distribution of the liposome-wall distance (which we determine from the mechanical properties of double-stranded-DNA chains). Our approach helps in deciphering the role of hydrodynamics in acoustic sensing and reveals the role of parameters hitherto largely unexplored. A practical consequence may be improvements in the design of biosensors and detection schemes.

DOI: 10.1103/PhysRevApplied.13.064059

\section{INTRODUCTION}

The quartz-crystal microbalance $(\mathrm{QCM})$ is an extremely versatile tool for studying soft matter at interfaces. QCMs cover a huge range of length scales (from nanometers to tens of micrometers), which makes them suitable for a myriad of applications ranging from nanotribology and health care to environmental monitoring [14]. In biophysics-related research, QCMs with dissipation monitoring (QCM-Ds) operate in liquids and follow

\footnotetext{
*rafael.delgado@uam.es
}

Published by the American Physical Society under the terms of the Creative Commons Attribution 4.0 International license. Further distribution of this work must maintain attribution to the author(s) and the published article's title, journal citation, and DOI. real-time changes in assemblies of lipid membranes, DNA, proteins, nanoparticles, viruses, and cells [4-9]. In addition, the QCM has become one of the important techniques for DNA sensing [10-12] and molecular sensing in general $[13,14]$, due to its low operating cost, the compactness of its sensor, the fact that it provides real-time data, and its subnanogram sensitivity. Generally, biosensing techniques rely on the principle of hybridization of the target (DNA), which binds to some probe fixed to a substrate. In a second hybridization step, the molecular target is decorated with a nanoparticle, whose role is to amplify the acoustic signal $[15,16]$. Notably, these "amplifiers" are often not adsorbed onto the resonator, but rather suspended in the liquid. Although most QCMbased biosensors deploy discrete suspended nanoparticles, their hydrodynamics has not so far been investigated theoretically. 
In a liquid, the transverse oscillation of the quartzcrystal resonator (typically between 5 and $65 \mathrm{MHz}$ ) creates a Stokes flow, which propagates momentum upwards to about 3 times the penetration depth $\delta=(2 \eta / \rho \omega)^{1 / 2}$ (between 250 and $70 \mathrm{~nm}$ in water). Here, $\omega=2 \pi f, \eta$ is the viscosity of the fluid, and $\rho$ is its density. The hydrodynamic stress created at the wall leads to a shift $\Delta f$ in the oscillator frequency and a shift $\Delta \Gamma$ in the decay rate (usually measured as the dissipation factor $D=2 \Gamma / f$ ). For the case of a (purely viscous) Newtonian fluid, $\Delta f$ and $\Delta D$ were derived by Kanazawa and Gordon [17] and in subsequent publications [18]. Johannsmann et al. [19] and Voinova et al. [20] later used effective-medium theory and phenomenological constitutive relations to estimate the viscoelastic properties of the assumed film formations of the material covering the sensor surface. However, viscoelastic film theories based on one-dimensional equations substantially fail to provide useful information in discreteparticle settings $[2,5]$. Experiments with adsorbed discrete particles such as liposomes and viruses made use of a different approach, providing estimations of the size of the nanoentities involved [9]. A model developed by Tsortos et al. $[7,21,22]$ allowed quantitative evaluation of the size and shape of tethered biomolecules (DNA and proteins) $[23,24]$; here, the hydrodynamic quantities of the intrinsic viscosity $[\eta]$ and the radius $R_{h}$ were explicitly taken into account and were linked to the acoustic ratio $\Delta D / \Delta f[21]$.

However, the problem of reducing the limit of detection in biosensors (which is still largely based on trial and error) requires finding the optimal acoustic amplification using nonadsorbed discrete particles. QCM sensors use acoustic enhancers such as magnetic beads $[25,26]$, gold nanoparticles [10-13], and, recently, liposomes [27] with sizes ranging from 10 to $100 \mathrm{~nm}$, which are often suspended in the liquid up to tens of nanometers away from the sensor surface. Theoretical understanding of such systems requires a detailed hydrodynamic analysis of the threedimensional unstationary flow patterns resulting from the propagation of fluid-induced forces acting on the analytes. In the case of adsorbed particles, numerical studies of QCM hydrodynamics qualitatively reproduce experimental observations, such as the coverage dependence of $\Delta D / \Delta f$, providing preliminary answers to a large list of still unexplained questions [2]. These studies [5,9], carried out in two dimensions using the commercial package COMSOL and, more recently, in three dimensions using lattice Boltzmann solvers [28,29], considered rigid particles adsorbed onto the wall at fixed positions (obstacles). However, fixing the particle position introduces ad hoc forces into the fluid (those required to keep the obstacle fixed), which surely alter the measurement of the system impedance. But, in the case of suspended particles, solving for the nanoparticle motion is essential. This requires taking into account the fluid traction and other forces acting on the analytes (elastic and interparticle forces, surface forces, contact forces, and advection due to a mean flow if required).

Using QCM biosensing to detect surface-adsorbed DNA with liposome amplifiers will probably have an impact on fast cancer diagnosis. Recent experiments have focused on the determination of the limit of detection of DNA [27] using this setup. Here we present a theoretical approach that is supported and guided by experimental data. Simulations are carried out with a finite-volume fluctuating hydrodynamic solver using the immersed boundary method, which describes the particle dynamics and uses an elasticnetwork model to reproduce the mechanical properties of molecules (e.g., bending rigidity). The good agreement between simulations and experiments [carried out with 1palmitoyl-2-oleoyl-sn-glycero-3-phosphocholine (POPC) liposomes] allows us to analyze the strong amplification of the acoustic signal, dominated by the perturbative flow of liposomes. The main parameters determining such amplification are the liposome radius $R$ and the distribution of the distances of the liposomes from the resonator, determined by the contour length of the DNA, $L_{\mathrm{DNA}}$.

\section{EXPERIMENTS}

Experiments are performed using a QCM-D setup (QSense E4, Sweden) based on the ring-down approach [30], which is capable of measuring changes in the resonance frequency $(\Delta f)$ and energy dissipation $(\Delta D)$ over time. In a QCM-D device, excitation pulses separated by milliseconds are sent to the piezoelectric material (a quartz crystal), inducing underdamped oscillations in the crystal, which are described by $x(t)=x_{0} \exp [-\Gamma t] \cos [2 \pi f t+\phi]$ (with $x_{0} \approx 2 \mathrm{~nm}$ ). The decay rate and frequency, $\Gamma$ and $f$, depend on the acoustic response related to the loading of the sensor. $\Gamma$ is often expressed in terms of the "dissipation factor" $D=2 \Gamma / f_{n}$. The fundamental frequency of the particular cut of the quartz crystal used is $f_{0}=5 \mathrm{MHz}$ (here we report experimental results for the seventh harmonic, $f_{7}=35 \mathrm{MHz}$ ).

The liposome-DNA ( $L+$ DNA) complexes that we use in this study (see the sketch at the top of Fig. 1) are formed by sequential injections of neutravidin, double-stranded DNA (dsDNA), and finally liposomes. Samples of dsDNA with 21,50 , and 157 base pairs (bp) (i.e., with lengths $L_{\text {DNA }}$ of 7 , 17 , and $53 \mathrm{~nm}$, respectively) are combined with liposomes of radii $R=15,25,50$, and $100 \mathrm{~nm}$. In order to be captured by the NAv layer previously formed on the surface (the probe), the target DNA fragments bear a biotin molecule at one end. In addition, a cholesterol molecule is incorporated on the opposite end of the DNA for subsequent liposome binding due to its strong affinity for the lipid membrane. Measurements are performed using a continuous flow rate of $60 \mu \mathrm{l} / \mathrm{min}$ and a fixed temperature of $25^{\circ} \mathrm{C}$. A more detailed description of the acoustic measurements is reported elsewhere [27]. Figure 1(a) shows a typical 


\section{(a)}
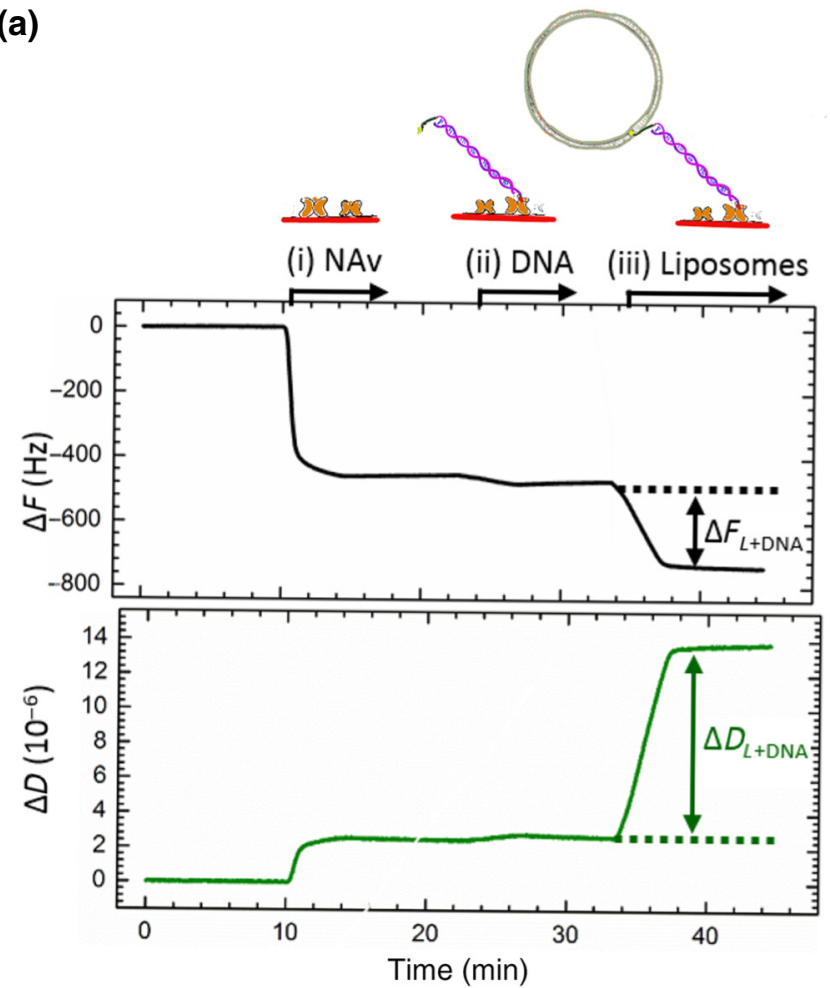

(b)

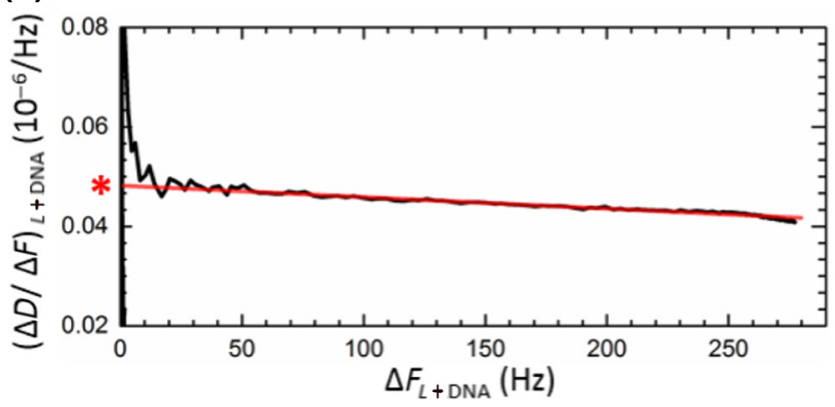

FIG. 1. (a) Representative sensogram depicting binding events recorded at $35 \mathrm{MHz}$ (i.e., seventh harmonic) in real time: (i) neutravidin (NAv) $(0.2 \mathrm{mg} / \mathrm{ml})$ is adsorbed onto a gold surface until saturation, followed by (ii) binding of biotinylated double-stranded DNA (here $50 \mathrm{n} M$ of 50 bp DNA, i.e., $17 \mathrm{~nm}$ long) bearing a cholesterol molecule at the opposite end; (iii) the latter can subsequently bind POPC liposomes (here $0.1 \mathrm{mg} / \mathrm{ml}$ of liposomes $25 \mathrm{~nm}$ in radius). The frequency and dissipation changes registered upon addition of liposomes are used to calculate the acoustic ratio of the liposome-DNA ( $L+\mathrm{DNA})$ complex as $\Delta D_{L+\mathrm{DNA}} / \Delta f_{L+\mathrm{DNA}}$. (b) Plot of the ratio $(\Delta D / \Delta f)_{L+\mathrm{DNA}}$ against the corresponding frequency shift (proportional to the liposome surface coverage) during the formation of the $L+$ DNA complex, between times of 32 and $38 \mathrm{~min}$ in (a). The dissipation capacity of the $L+$ DNA complex is marked with an asterisk (see text).

sensogram depicting the formation of an $L+$ DNA complex in real time. The figure illustrates the changes in the $\Delta f$ (black) and $\Delta D$ (green) signals recorded upon sequential injections of (i) NAv, (ii) DNA, and (iii) POPC liposomes.
The plateau values corresponding to the frequency and dissipation changes registered after addition of liposomes are used to calculate the acoustic ratio of the $L+$ DNA complex as $\Delta D_{L+\mathrm{DNA}} / \Delta f_{L+\mathrm{DNA}}$. Figure $1(\mathrm{~b})$ plots the acoustic ratio $r_{\text {acoustic }} \equiv-(\Delta D / \Delta f)_{L+\mathrm{DNA}}$ against $\Delta f_{L+\mathrm{DNA}}$ for all points of the sensogram, starting from the onset of regime (iii) in Fig. 1(a) [from 32 to $38 \mathrm{~min}$ in Fig. 1(a)]. The dissipation capacity $C_{d}$ of the $L+$ DNA complexes [indicated by an asterisk in Fig. 1(b)] is obtained by a standard procedure, where $C_{d}$ is taken as the intercept at $\Delta f_{L+\mathrm{DNA}}=$ 0 of a linear fit to the acoustic ratio against $\Delta f$ (red line). All reported measurements of $\Delta f$ are used in the form of raw data, i.e., without dividing them by the number of the harmonic.

\section{THEORETICAL AND NUMERICAL ANALYSIS}

\section{A. Impedance}

Our theoretical and numerical analysis is based on the well-established small-load approximation (SLA) [2], which relates the impedance $\mathcal{Z}=\hat{\sigma} / v_{0}$ (the ratio of the wall stress to the surface velocity) to the complex frequency shift $\Delta \tilde{f}=\Delta f+i \Delta \Gamma$ measured in ringdown experiments. The SLA is valid if the resonator's mass per unit area is much larger than the load, which is a safe approximation in our case (where $\Delta f / f_{0}<$ $10^{-5}$ ). The impedance of the complex load is expressed as the sum $\mathcal{Z}=\mathcal{Z}_{Q}+\mathcal{Z}_{0}+\mathcal{Z}_{\mathrm{DNA}}+\mathcal{Z}_{L+\mathrm{DNA}}$, where the impedances correspond to the clean quartz resonator $\left(\mathcal{Z}_{Q}\right)$, the (unloaded) Newtonian solvent $\left(\mathcal{Z}_{0}\right)$ [17], the DNA strand without a liposome $\left(\mathcal{Z}_{\mathrm{DNA}}\right)$, and the $L+\mathrm{DNA}$ complex $\left(\mathcal{Z}_{L+\text { DNA }}\right)$, respectively. For any contribution (different from $Q$ ), the SLA yields [2]

$$
\Delta f_{a}+i \Delta \Gamma_{a}=i f_{0} \frac{\mathcal{Z}_{a}}{\pi \mathcal{Z}_{Q}},
$$

where $\mathcal{Z}_{Q}=8.8 \times 10^{6} \mathrm{~kg} /\left(\mathrm{m}^{2} \mathrm{~s}\right)$, and we recall that the dissipation shift is given by $\Delta D=2 \Delta \Gamma / f_{n}$. The real part of $\mathcal{Z}_{a}$ is related to the dissipation and the imaginary part to the frequency shift $\left(\operatorname{Re}\left[\mathcal{Z}_{a}\right] \propto-\Delta \Gamma\right.$ and $\left.\operatorname{Im}\left[\mathcal{Z}_{a}\right] \propto-\Delta f\right)$, while $r_{\text {acoustic } a}=-2 \operatorname{Re}\left[\mathcal{Z}_{a}\right] /\left(\operatorname{Im}\left[\mathcal{Z}_{a}\right] f_{n}[\mathrm{MHz}]\right)$, and $f_{n}$ is the working frequency ( $n=7$ in Fig. 1$)$.

\section{B. Simulations by the immersed boundary method}

Our mesoscopic model is based on a fluctuating hydrodynamic solver for compressible unsteady flow that uses the immersed boundary method to couple the dynamics of the fluid and the structure [31-33]. It is implemented in the graphics-processor-unit (GPU) code FLUAM ("FLuid And Matter interaction"), a second-order-accurate finitevolume scheme on a staggered grid [34] of side $h$. The liposome and dsDNA are represented using beads of radius $h$ (Fig. 2) connected by harmonic springs and/or bending potentials (see Sec. A 3). An elastic network is used 
to model the membrane of the (hollow) liposome by connecting nearest neighbors of the network with harmonic springs: the expression for the bonding force $\mathbf{F}_{i j}=$ $-k_{L}\left(\mathbf{r}_{i j}-r_{0}\right)$ includes the equilibrium distance $r_{0} \approx h$ and the spring constant $k_{L}$ determining the rigidity of the liposome. Here we consider the rigid limit (high $k_{L}$ ) to focus on the leading contribution to the impedance. The dsDNA is modeled by a bead model for semiflexible polymers, with the bending energy extracted from the DNA persistence length at room temperature $(50 \mathrm{~nm})$. We use the term "link" to denote the DNA-wall force $F_{\text {link }}$.

The geometry is illustrated in Fig. 2. The simulation box is periodic in the resonator $x-z$ plane, with dimensions $L \times L_{y} \times L$. Rigid no-slip walls at $y=0$ and $y=L_{y}$ are imposed via explicit boundary conditions $[31,35]$. The top wall is kept at rest, while the bottom wall, at $y=0$, moves in the $x$ direction with velocity $v_{\text {wall }}=v_{0} \cos (2 \pi f t)$ with $v_{0}$ set to fix a small wall displacement $x_{0}<h$. To achieve the required numerical convergence, we use a spatial resolution of $h=3.958 \mathrm{~nm}$ (see Fig. 9 in Appendix B). The code units map the density and kinematic viscosity of water at $T=25^{\circ} \mathrm{C}$. For more details, see Table I and Appendix A. The sound velocity $c$ is set to match the experimental value of the nondimensional group $f_{7} \delta / c \sim 3 \times 10^{-3}$, whose smallness indicates a minor effect of fluid compressibility [36]. Moreover, the large time-scale separation

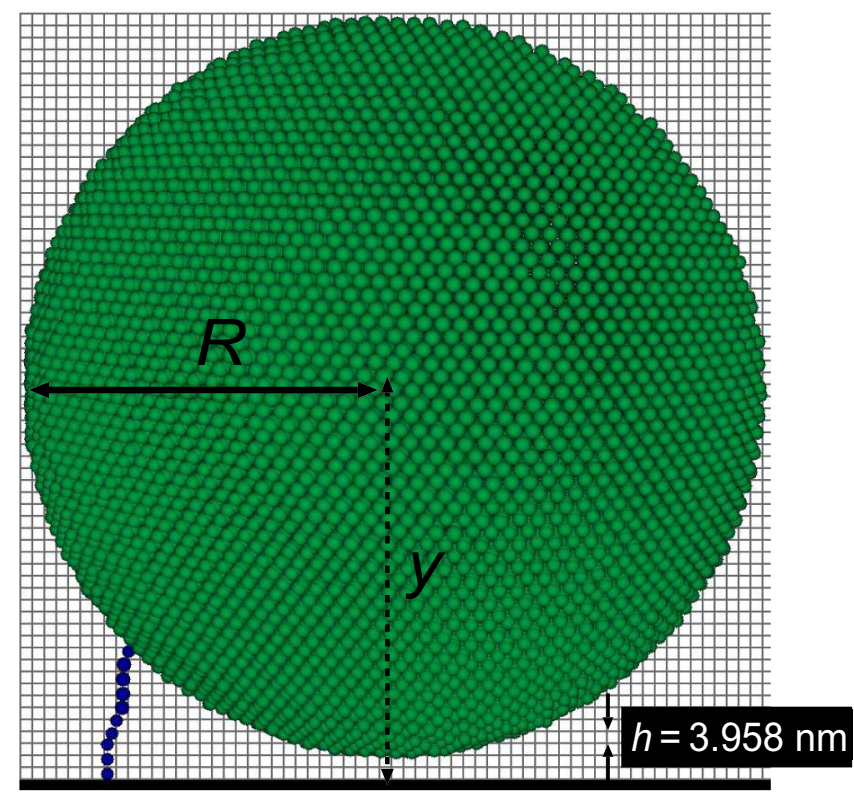

FIG. 2. Snapshot of the numerical model of a liposome of radius $R=100 \mathrm{~nm}$ tethered to a DNA strand attached to the resonator wall (see text). The coupling between the dynamics of the analyte and the fluid is solved for using the immersed boundary method [31,33]. The spatial resolution is set by the fluid cell size $h$ (here $3.958 \mathrm{~nm}$ ). The bottom wall oscillates transversely at $35 \mathrm{MHz}$, with a small amplitude (less than one cell).
TABLE I. Relation between code units and SI units, code and SI values of the parameters used in the simulations, and equivalences between real and imaginary impedances $Z$ (code units) and frequency shifts $\Delta f(\mathrm{~Hz})$ and dissipation factor $\Delta D$ for the seventh harmonic of a quartz crystal with fundamental frequency $f_{0}=5 \mathrm{MHz}$.

\begin{tabular}{lcc}
\hline \hline Magnitude & Code units & SI units \\
\hline Length & 1 & $7.917 \mathrm{~nm}$ \\
Mass & 1 & $4.96 \times 10^{-22} \mathrm{~kg}$ \\
Time & 1 & $1.416 \times 10^{-11} \mathrm{~s}$ \\
Kinematic viscosity & 0.226 & $10^{-6} \mathrm{~m}^{2} / \mathrm{s}$ \\
Fluid density & 1 & $10^{3} \mathrm{~kg} / \mathrm{m}^{3}$ \\
Sound velocity & 2.68 & $1.5 \times 10^{3} \mathrm{~m} / \mathrm{s}$ \\
QCM frequency & 0.0005 & $35 \mathrm{MHz}$ \\
$k_{B} T$ & $6.6 \times 10^{-5}$ & $4 \times 10^{-21} \mathrm{~J}$ \\
$Z_{Q}$ & 7.925 & $8.8 \times 10^{6} \mathrm{~kg} /\left(\mathrm{m}^{2} \mathrm{~s}\right)$ \\
$\operatorname{Im}[Z]$ & $10^{-3}$ & $\Delta f=200.8 \mathrm{~Hz}$ \\
$\operatorname{Re}[Z]$ & $10^{-3}$ & $\Delta D=1.826 \times 10^{-6}$ \\
\hline \hline
\end{tabular}

between the liposome diffusion time and the QCM oscillation period $\left(6 \pi \eta R^{3} f / k_{B} T>10^{4}\right)$ makes it possible to neglect thermal fluctuations over the short simulation runs we use to evaluate the impedance (between 10 and 20 periods). We stress, however, that in our simulations the liposome is free to move according to the fluid traction; this is essential to obtain unbiased results, particularly because the liposomes are not adsorbed but suspended.

\section{Computational protocol}

"Instantaneous" values of $f$ and $D$ in a QCM experiment correspond to an ensemble average over many $L+$ DNA assemblies and a large number of sequences of pulse-waiting times (each one in the millisecond range). As a direct route to the average impedance, we prepare a set of 40 initial equilibrium configurations using Monte Carlo (MC) sampling of the $L+$ DNA complex (see Appendix A for details of the MC sampling) to obtain the average impedance. To that end, the impedance of each configuration is measured by running FLUAM over ten periods, after a transient regime (typically two periods). We record the time-dependent wall stress averaged over the resonator plane, $\sigma(t)$, which is decomposed as $\sigma(t)=\sigma_{\text {hydro }}+\sigma_{\text {link }}$, where $\sigma_{\text {link }}=F_{\text {link }} / L^{2}$ is the contribution of the DNA-wall link, while the contribution of the hydrodynamic stress is $\sigma_{\text {hydro }}(t)=\eta\left\langle\left[\partial v_{x}(\mathbf{r}, t) / \partial y\right]_{\text {wall }}\right\rangle$ (angle brackets denote an average over the whole surface). Setting $\sigma(t)=\operatorname{Re}[\hat{\sigma} \exp (-i \omega t)]$ leads to the stress phasor $\hat{\sigma}$ after fitting $\sigma(t)=\operatorname{Re}[\hat{\sigma}] \cos (\omega t)+\operatorname{Im}[\hat{\sigma}] \sin (\omega t)$. This provides the total impedance $\mathcal{Z}=\hat{\sigma} / v_{0}$ of each initial configuration. The impedance of the $L+$ DNA complex is $\mathcal{Z}_{L+\mathrm{DNA}}=\mathcal{Z}-\mathcal{Z}_{0}-\mathcal{Z}_{\mathrm{DNA}}$, where $\mathcal{Z}_{0}=(i-1) \eta / \delta$ corresponds to the unperturbed (Stokes) flow and $\mathcal{Z}_{\mathrm{DNA}}$ to the DNA anchor (without liposome), which is found to be negligible (see below). 
Our calculations of $\mathcal{Z}_{L+\text { DNA }}$ are carried out for increasing box side $L$ (i.e., a surface density $\phi=1 / L^{2}$ ) using periodic boundary conditions in the resonator plane. This corresponds to a periodic array of analytes, which differs from the experimental randomness. However, the relation between $\Delta D$ and $\Delta f$ is similar to what is observed in experiments. Such a relation is illustrated in Fig. 3, which compares simulated and experimental values of $\Delta D$ against $\Delta f$ (we also include the liposome surface fraction $\phi$ obtained from the simulations). The simulations closely follow the experimental trend: at low coverage, a linear relation $\Delta D \approx C_{d} \Delta f$ determines the dissipation capacity. A transition to a sublinear regime $\Delta D \sim(\Delta f)^{0.8}$ takes place above a certain coverage (approximately 0.12 in Fig. 3). Such a transition has also been reported in other experiments [2]. Although a clear theoretical explanation is still lacking, this subtle coverage effect (which induces a decrease in the acoustic ratio with $\phi$ ) arises from hydrodynamic interactions between large particles (liposomes or viruses). The effect is absent in unloaded DNA strands, a fact that is confirmed by our simulations (see Appendix C and Fig. 10), and it has also been experimentally observed [21]. The effect is also absent in the case of DNA-protein assemblies [37].

To strictly follow the experimental procedure, we would need to extract the contribution of the bare DNA anchor $\mathcal{Z}_{\text {DNA }}$ from the total impedance to obtain the liposomeDNA impedance, i.e., $\mathcal{Z}_{L+\text { DNA }}=\mathcal{Z}-\mathcal{Z}_{0}-\mathcal{Z}_{\text {DNA }}$. However, similarly to what happens in the experiments, the impedance of bare DNA at such low concentrations is
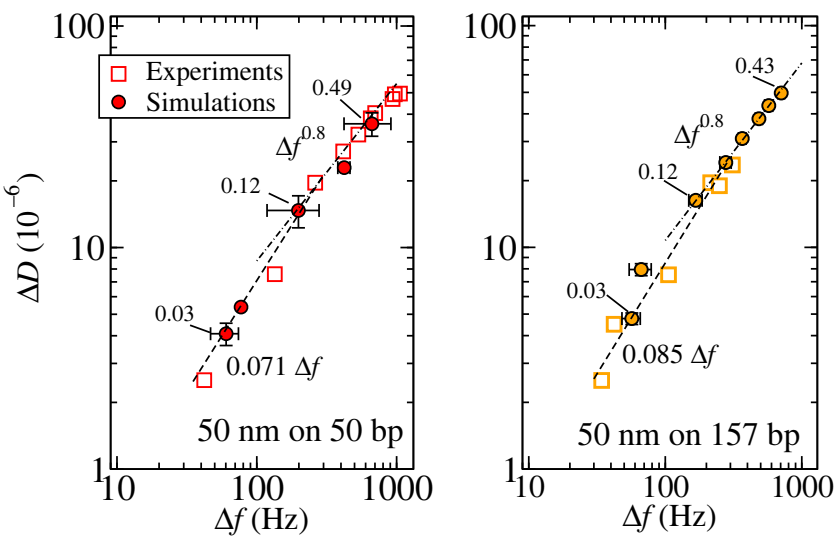

FIG. 3. Dissipation versus frequency shift obtained in experiments and simulations for liposomes of radius $R=50 \mathrm{~nm}$ tethered to DNA strands of length $50 \mathrm{bp}\left(L_{\mathrm{DNA}}=17 \mathrm{~nm}\right)$ (left panel) and $157 \mathrm{bp}\left(L_{\mathrm{DNA}}=53 \mathrm{~nm}\right)$ (right panel). The numbers above the simulation data indicate the liposome occupation fraction $\mu \equiv \pi R^{2} \phi$, where $\phi$ is the coverage (number of liposomes per unit area). Dashed lines indicate the linear relation $\Delta D=C_{d} \Delta f$ used to obtain $C_{d}$. Dot-dashed lines indicate the sublinear regime $\Delta D \sim(\Delta f)^{0.8}$, which arises due to hydrodynamic interaction among liposomes. often too small to be numerically detected in simulations. This is precisely the role of the amplifier. For a similar surface coverage, we estimate that the liposome amplifies the acoustic signal of the bare DNA by between 2 and 4 orders of magnitude (depending on $R$ and $L_{\mathrm{DNA}}$ ). For a further consistency check, we increase the concentration of bare DNA in the simulations and, as stated, we confirm that the scaled DNA impedance $\mathcal{Z}_{\mathrm{DNA}} / \phi$ is constant (see Appendix $\mathrm{C}$ for an analysis of unloaded DNA). This indicates that hydrodynamic interactions between bare DNA strands are negligible even at relatively high concentrations.

\section{RESULTS AND DISCUSSION}

Numerical and experimental estimations of the dissipation capacity $C_{d}=C_{d} L+$ DNA are compared in Fig. 4. An increase in $C_{d}$ with the liposome radius $R$ and the DNA contour length $L_{\mathrm{DNA}}$ is observed both in the simulations and in the experiments. The agreement is quite close to being quantitative and gives us confidence to deploy our model to analyze some of the large number of factors affecting the value of $C_{d}$. Such a task demands a theoretical analysis of the impedance.

\section{A. Analysis}

The impedance of the $L+$ DNA assembly can be decomposed into contributions from the liposome $(L)$ and the DNA, namely, $\mathcal{Z}_{L+\mathrm{DNA}}=\mathcal{Z}_{L+\mathrm{DNA}}^{(\mathrm{L})}+\mathcal{Z}_{L+\mathrm{DNA}}^{\text {(DNA })}$. The term $\mathcal{Z}_{L+\mathrm{DNA}}^{(\mathrm{DNA})}$ collects together the contributions of the linker and the hydrodynamic impedance of the DNA. Note that with the exception of the small molecular linker (biotin),

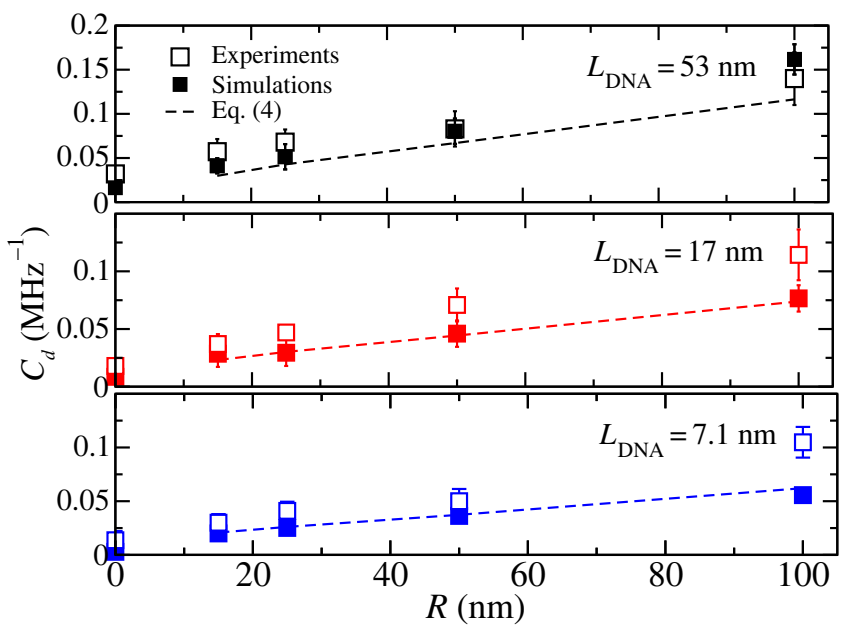

FIG. 4. Dissipation capacity of liposome-DNA complexes at a frequency $f_{n}=35 \mathrm{MHz}$, plotted against the liposome radius $R$ for different dsDNA contour lengths. Filled symbols correspond to simulation results, and open symbols to experiments using POPC liposomes at $T=25^{\circ} \mathrm{C}$ [27]. The simulations are performed with a mesh with $h=3.958 \mathrm{~nm}$ in boxes of side $L=506.6 \mathrm{~nm}$. The dashed lines correspond to Eq. (4) [using Eq. (2) for $\mathcal{Z}_{L}$ ]. 
the DNA chain is suspended in the fluid, and the dominant part of its impedance is of hydrodynamic origin. In general, the hydrodynamic impedance arises from fluidinduced forces (or force distributions) on the solutes, which are transferred back to the fluid by momentum conservation and propagate by viscous diffusion to the resonator, creating stress on the wall. The lack of symmetry makes an analytical approach to this fluid-induced dynamics elusive, even for a point particle in a Stokes flow [38]. The fluid-induced forces could be of inertial origin (relative fluid-particle accelerations leading to a net force on the solute). However, due to the extremely small density contrast of the quasi-neutrally buoyant liposomes and DNA, inertia is negligible. Even so, the liposomes will have a stress distribution at their surface due to their reaction against flow-induced deformation. The liposome surface stress (in addition to the line tension along the DNA) is propagated back to the wall by viscous transport. There, at the resonator, the only way to disentangle $\mathcal{Z}_{L+\mathrm{DNA}}^{(\mathrm{L})}$ from $\mathcal{Z}_{L+\mathrm{DNA}}^{(\mathrm{DNA})}$ is to compare the impedance of the $L+$ DNA assembly with that of a hypothetical free-floating (not tethered) liposome $\mathcal{Z}_{L}$ placed at a similar height $y$. This comparison, shown in Fig. 5, shows that, at least for $R>15 \mathrm{~nm}$, the dominant contribution to $\mathcal{Z}_{L+\text { DNA }}$ comes from the liposome surface stress, determined by $\mathcal{Z}_{L}(y)$.
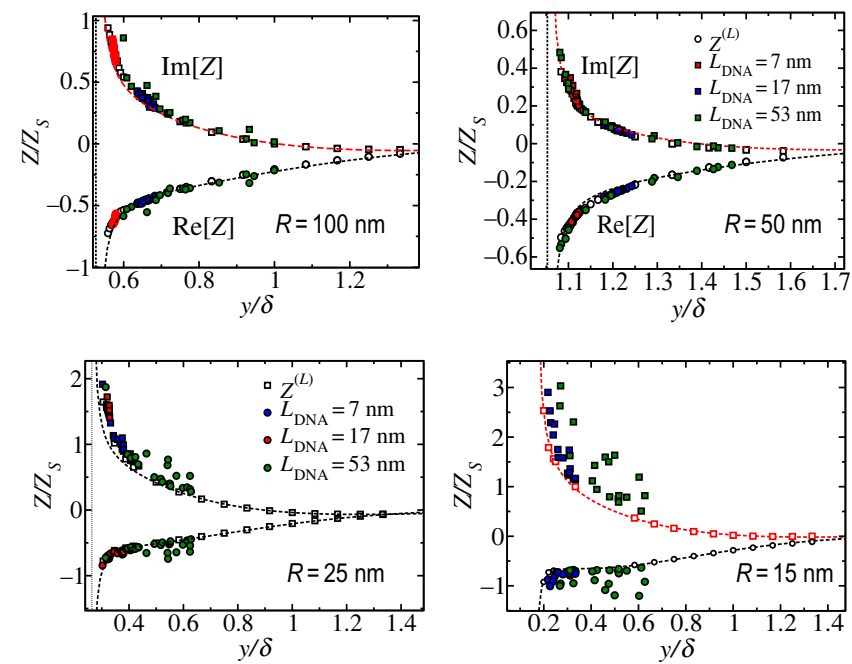

FIG. 5. Acoustic impedance $\mathcal{Z}_{L+\text { DNA }}$ of the liposomeDNA assembly scaled by the stresslet impedance $Z_{S}=$ $(20 \pi / 3) R^{3} \eta /\left(\delta^{2} L^{2}\right)$ for (top) liposome radius $R=100$ and $50 \mathrm{~nm}$ and (bottom) 25 and $15 \mathrm{~nm}$. Lines and open circles correspond to numerical results and the theoretical trend [Eq. (2)], respectively, for $\mathcal{Z}_{L}$ for a freely suspended liposome, and filled symbols correspond to $\mathcal{Z}_{L+\text { DNA }}$ for individual configurations of the liposome-DNA complex and several DNA contour lengths. The vertical line indicates the wall. Results are obtained for $f_{7}=35 \mathrm{MHz}$ (for which $\delta=95 \mathrm{~nm}$ ) in square boxes of side $506.67 \mathrm{~nm}$ (and $L_{x}=L_{z}=203.33 \mathrm{~nm}$ in the cases of $R=15$ and $25 \mathrm{~nm}$ ).
Before analyzing the role of the DNA strand, we focus on $\mathcal{Z}_{L}(y)$ in Fig. 5. Remarkably, $\mathcal{Z}_{L}(y)$ decays almost exponentially with $y$ : this general trend can be explained by hydrodynamic reflection. Resonator vibrations (with velocity $v_{0}$ ) are propagated upwards to the fluid by viscous forces and create an unsteady and distance-dependent Stokes flow. The velocity field (phasor) $v_{0} \exp [-\alpha y]$ contains the viscous propagator $\exp [-\alpha y]$, with $\alpha=$ $(1-i) / \delta$. The behavior of $\mathcal{Z}_{L}(y)$ can be understood using a corollary of Faxén's theorem (valid for steady [39] and unsteady [40] flow), which guarantees that the solute surface stress is a linear function of the ambient flow. Far away from the resonator, the ambient flow is similar to the Stokes flow, and so the liposome surface stress should vary with the vertical coordinate as $\exp [-y \alpha]$. Viscous propagation propagates this flow-induced liposome surface stress back to the surface. This reflection brings another factor $\exp [-y \alpha]$ to the wall stress, leading to $\mathcal{Z}_{L} \sim$ $Z_{S} \exp [-2 \alpha y]$. The prefactor $Z_{S}=(20 \pi / 3) \eta R^{3} /\left(L^{2} \delta^{2}\right)$ is chosen to be consistent with the stresslet of a sphere under steady shear. In particular, the local stresslet in a steady linear flow is $\mathcal{S}=(20 / 3) \pi \eta R^{3}\left[1+\left(R^{2} / 10\right) \nabla^{2}\right] \mathcal{E}^{(0)}$, where the tangential strain is $\mathcal{E}_{x y}^{(0)}=(1 / 2) d v_{x}^{(0)} / d y$. If the sphere is not close to the resonator, we thus expect the impedance to be of the form $Z_{S}\left[a(\alpha R)^{2}+b(\alpha R)\right] \exp [-2 \alpha y]$, where $(\alpha R)^{2}=-2 i(R / \delta)^{2}, a$ is some constant, and $b(\alpha R)$ is some function. Close to the resonator, the ambient flow includes a significant contribution from the wall reaction field [41]. As the particle approaches the surface, multiple hydrodynamic reflections between the wall and the particle create a strong perturbative field. This hydrodynamic feedback, which has been studied asymptotically for point particles [38] (in relation to Brownian motion), induces a large increase in the impedance. From the asymptotic behavior of the wall reaction flow of a point particle [41], we expect that the near-field contribution to the impedance scales roughly as the inverse of the surface-to-sphere distance $y-R$. This leads us to the following ansatz:

$$
\mathcal{Z}_{L}(y)=Z_{S}\left[(A+i B) \exp [-2 \alpha y]+\frac{2 i C}{\alpha(y-R)}\right]
$$

This ansatz nicely recovers the simulation results for all particle sizes, as shown in Fig. 5. Numericalsimulation data suggest the following trends for the parameters [42]: $A \approx 1.40(R / \delta)^{2}, B \approx 1.6-0.1 \exp \left[(R / \delta)^{2}\right]$, and $2 C \approx 0.01(1+i)+(1-i) f(R / \delta)$, with $f(R / \delta)=$ $0.035 \exp (-R / \delta)$ (see Sec. C 2 and Fig. 11 for details of the fitting parameters). We show later that using this set of parameters in Eq. (2) satisfactorily reproduces the value of $C_{d}$ for different forcing frequencies.

The contribution of the DNA can now be estimated as $\mathcal{Z}_{L+\mathrm{DNA}}^{(\mathrm{DNA})}=\mathcal{Z}_{L+\mathrm{DNA}}-\mathcal{Z}_{L}$. As shown in Fig. 12 , we find 
that

$$
\mathcal{Z}_{L+\mathrm{DNA}}^{\text {(DNA) }} \approx\left(-7.2 R^{-2}+i 83 R^{-2.5}\right) L_{\mathrm{DNA}}^{0.5} Z_{S},
$$

where $R$ and $L_{\mathrm{DNA}}$ are in nanometers. For $R>15 \mathrm{~nm}$, this represents a minor contribution to the total impedance $\mathcal{Z}_{L+\mathrm{DNA}}$, but for $R<15 \mathrm{~nm}$ it becomes noticeable (see Fig. 5 and Fig. 12 in Sec. C 3 for an analysis of the DNA contribution). As an aside, we note that the impedance related to the loaded DNA is much larger than that of the bare chain, i.e., $\mathcal{Z}_{L+\mathrm{DNA}}^{(\mathrm{DNA})} \gg \mathcal{Z}_{\mathrm{DNA}}$. This is due to the extra line tension arising from the forces induced by the DNA being constrained to the suspended liposome.

Figure 5 shows that the dispersion of $\mathcal{Z}_{L+\mathrm{DNA}}$ around its average decaying trend is relatively small and does not greatly vary with $y$. Thus $\mathcal{Z}_{L+\mathrm{DNA}}^{\text {(DNA) }}$ does not vary strongly with the orientation of the DNA strand. In terms of the acoustic response, the most important effect of the DNA is to constrain the liposome position and determine the liposome height distribution $P(y)$. In general, the average impedance can be obtained from the weighted average

$$
\left\langle\mathcal{Z}_{L+\mathrm{DNA}}\right\rangle=\int P(y) \mathcal{Z}_{L+\mathrm{DNA}}(y) d y,
$$

where $\mathcal{Z}_{L+\mathrm{DNA}}(y)=\mathcal{Z}_{L}(y)+\mathcal{Z}_{L+\mathrm{DNA}}^{\text {(DNA) }}$. The relation in Eq. (4) is extremely useful because it decomposes the analyte and anchor contributions, allowing fast evaluation of nontrivial effects. $P(y)$ encodes important microscopic information about the anchor: the bending rigidity, the linker-DNA tilt energy [43], or large values of the DNA coverage [44], which could lead to multiple anchors connected to the liposome [27]). In general, $P(y)$ can also introduce information into Eq. (4) about physicochemical forces between the analyte and the wall (solvation, dispersion, electrostatic forces), as well as the effect of advection under a strong Poiseuille flow. All these effects are known to alter the acoustic ratio, and their relevance can be tested using Eq. (4), by pre-evaluating $P(y)$, either theoretically or from Monte Carlo simulations. We use MC sampling to obtain $P(y)$ for an electrically neutral POPC liposome anchored by a single DNA chain. Figure 6 compares the numerically estimated $P(y)$ with a theoretical trend derived by assuming that the short dsDNA strands are almost rigid and by setting a maximum allowed angle $\theta_{\max }$ between the normal to the liposome surface and the DNA strand. Elementary trigonometric relations (see the caption of Fig. 6) lead to

$$
P(y)=\left\{\begin{array}{cl}
C^{-1}\left(R_{2}^{2}-R_{1}^{2}\right) & y<R_{1}, \\
C^{-1}\left(R_{2}^{2}-y^{2}\right) & R_{1} \leq y \leq R_{2}, \\
0 & y>R_{2}
\end{array}\right.
$$

with (a)
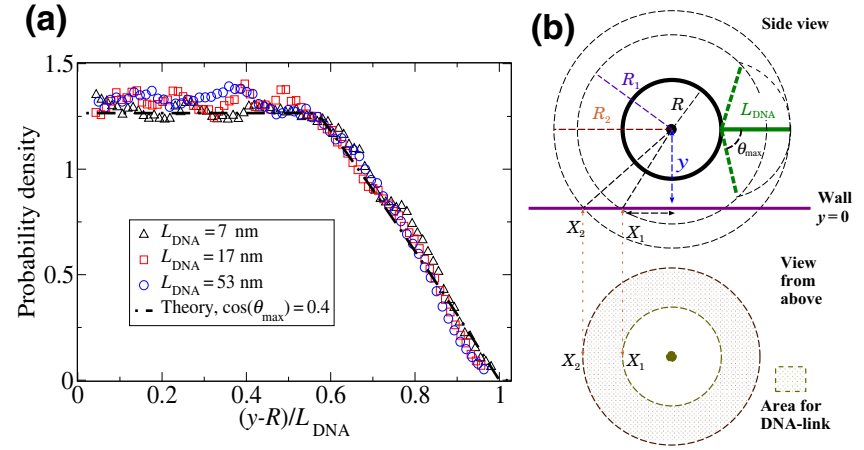

FIG. 6. (a) Monte Carlo results for the probability density of finding a liposome of radius $R$ tethered to a dsDNA strand of length $L_{\mathrm{DNA}}$ at height $y$. The scaled length $(y-R) / L_{\mathrm{DNA}}$ provides a master curve for all values of $L_{\mathrm{DNA}}$ (the results correspond to $R=100 \mathrm{~nm}$ ). The dashed line corresponds to the theoretical estimation in Eq. (5) with $\cos \left(\theta_{\max }\right)=0.4$. (b) Sketch indicating the theoretical derivation: $P(y)$ is proportional to the dashed area (see "View from above"), where the DNA strand (green lines) is able to contact the wall, starting from any position at the liposome surface, and provided that the liposome center is at a height $y$ above the wall. The region accessible to the end of the DNA is enclosed between spheres of radii $R_{1}$ and $R_{2}$. The outer radius is $R_{2}=R+L_{\mathrm{DNA}}$ (corresponding to $\theta=0$ ), while $R_{1}$ [in Eq. (6)] corresponds to $\theta=\theta_{\max }$ (the maximum allowed tilt of the DNA).

$$
\begin{aligned}
R_{1} \equiv & {\left[R^{2}+L_{\mathrm{DNA}}^{2}+2 L_{\mathrm{DNA}} R \cos \left(\theta_{\mathrm{max}}\right)\right]^{1 / 2}, } \\
R_{2} \equiv & R+L_{\mathrm{DNA}}, \\
C \equiv & \left(R_{2}^{2}-R_{1}^{2}\right)\left(R_{1}-R\right)+\left(R_{2}-R_{1}\right)\left(R+L_{\mathrm{DNA}}\right)^{2} \\
& -\frac{1}{3}\left(R_{2}^{3}-R_{1}^{3}\right) .
\end{aligned}
$$

The numerical results for $P(y)$ are in excellent agreement with Eq. (5) using $\theta_{\max } \approx 66^{\circ}$ (see Fig. 6). In the liposomeDNA bead model (see Fig. 2), the steric repulsion between the DNA and the liposome bead prevents the DNA from tilting by more than $\theta_{\max }$. Although we do not have an estimation of the experimental value of $\theta_{\max }$, a similar steric limitation on the tilt angle might also arise. In practice, varying the limiting angle has a small effect on the acoustic ratio.

By substituting $P(y)$ into Eq. (4) and using $\mathcal{Z}_{L}$ in Eq. (2) and $\mathcal{Z}_{\mathrm{DNA}}^{(\mathrm{DNA})}$ in Eq. (3), we obtain values of $C_{d}$ (dashed lines in Fig. 4) that are consistent with the direct numerical sampling and quite close to the experimental results. Finally, it is important to validate the simulations and the analytical approximations to the impedance against the forcing frequency of the QCM. To this end, we gather experimental data for different harmonics of the quartz-crystal resonator and, in Fig. 7, compare experimental values for $C_{d}$ with simulation results at different frequencies $f_{n}$. Results obtained using Eqs. (4) and (2) are also included. 


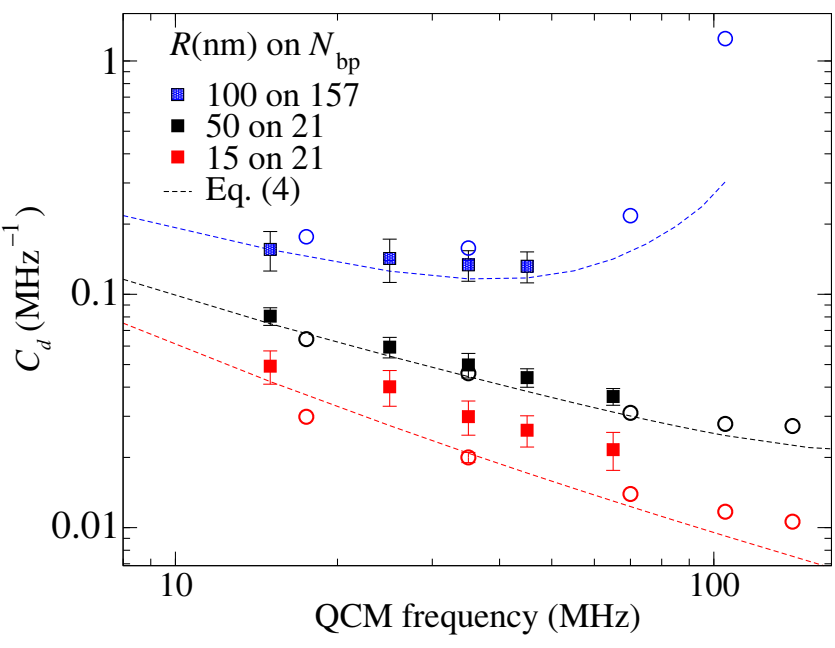

FIG. 7. Dissipation capacity $C_{d}$ [i.e., the linear extrapolation of $(\Delta D / \Delta f)$ to $\Delta f=0$ ] plotted against the forcing frequency $f_{n}$ of the QCM for several combinations of liposomes of radius $R$ and DNA strands with $N_{\mathrm{bp}}$ base pairs [the notation used in the figure is " $R(\mathrm{~nm})$ on $N_{\mathrm{bp}}$ "]. Squares correspond to experimental values of $C_{d}$ for different harmonics $f_{n}$, and circles correspond to simulations. The dashed lines are obtained from Eqs. (4) and (2).

The overall agreement is quite good, both in the values and in the trends. We find that the value of $C_{d}$ obtained from the simulations is somewhat smaller than the experimental values (see also Fig. 4). We discuss the possible physical origins of this discrepancy in the next section. In any case, it is important to stress the large sensitivity of the acoustic ratio to very small variations in the impedance. This sensitivity is particularly high for the largest particles on the largest DNA strands because the frequency shift (the imaginary part of the impedance) approaches zero (and thus $C_{d}$ approaches a divergence; see Fig. 7). To further illustrate this point, we plot the value of $C_{d}$

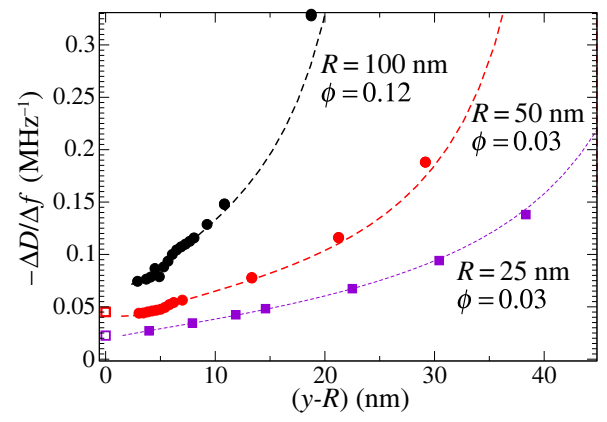

FIG. 8. Dissipation capacity $C_{d}$ (i.e., $-2 \operatorname{Re}\left[\mathcal{Z}_{L}\right] / \operatorname{Im}\left[\mathcal{Z}_{L}\right] / f_{n}$ at low $\phi$ ) of free particles of radius $R$ whose center is at a height $y$ above the resonator. The coverage fraction $\phi=\pi(R / L)^{2}$ is also indicated. The results are plotted against the gap size $y-R$, so that squares correspond to particles on the wall. The dashed lines come from Eq. (2). The results correspond to $f_{n}=35 \mathrm{MHz}$. for free-floating particles located at some given height $y$ against the surface-to-surface gap $y-R$ in Fig. 8. Notably, although the impedance $Z$ decreases exponentially with $y$ (see Fig 5), the acoustic ratio increases with $y$ and eventually diverges at some height where $\operatorname{Im}[Z](y)$ vanishes. The existence of a "zero-frequency crossing" due to a mass being located at some "critical" height above the resonator is in fact a quite general hydrodynamic feature [45]. This effect leads to a radically different behavior of $C_{d}$ for suspended particles compared with adsorbed ones (Fig. 8).

\section{CONCLUSIONS}

By combining experiments, theory, and simulations, we analyze the QCM response of liposome-DNA complexes used for acoustic amplification in DNA sensing. The liposomes are anchored to dsDNA strands and suspended in a fluid solvent. In general terms, our setup can be seen as an archetype of QCM molecular sensors, where other types of suspended nanoparticles are used as amplifiers. The procedure and many of the conclusions of the work presented here can be extrapolated to other devices (e.g., based on gold nanoparticles [10-12]). We show that the acoustic response of a suspended particle depends strongly on its height distribution $P(y)$. The phenomenon of hydrodynamic reflection [39] creates feedback between the perturbative flow induced by the particle and the hydrodynamic response of the wall. When the nanoparticle is close to the wall, such feedback leads to a large amplification of the impedance, which becomes about 3 orders of magnitude larger than that of the bare DNA chain. The problem of a suspended nanoparticle moving under fluidinduced forces close to a high-frequency oscillatory wall is at the boundary of current knowledge in the field of zeroReynolds-number theoretical hydrodynamics and has not been analytically solved [38,40,41]. Analytical approaches have so far been focused on point particles (hydrodynamic monopoles). But acoustic amplifiers are typically of the same size as the fluid penetration depth (about $100 \mathrm{~nm}$ ), and their description requires higher moments of the fluid traction [39]. Notably, liposomes are neutrally buoyant, and so the leading term is the stresslet (the second-order moment).

In this paper, we deploy the immersed boundary method to solve for the fluid-structure coupling, combined with an elastic-network model to model the macromolecular complexes. Combining both techniques is particularly useful for QCM research on soft matter. Deciphering the complicated hydrodynamics behind discrete-particle QCM experiments is key not only to a theoretical understanding but also to controlling its applications. The QCM senses the extra stress at the resonator arising from the perturbative flow created by the analyte. And, in turn, this perturbative flow is activated by fluid-induced forces on the analyte. 
Here we consider liposomes [27]. Although they are neutrally buoyant (force-free), we prove that liposomes are highly dissipative due to the viscous propagation of their surface stress to the wall. A possible extension of this work would be to consider heavy particles with inertia, and also to include other subtle mechanisms that alter the response of liposomes, such as their bending rigidity $\kappa$ and the fluidity of the lipid membrane. In a set of preliminary calculations, we decrease the bending rigidity of the liposome model, by reducing the spring constant $k_{L}$ used to connect the elastic network forming the liposome model. Reducing $k_{L}$ from 100 (rigid limit) to $k_{L}=10$ (flexible) (which corresponds to a reduction of the elastic modulus by a factor of 10) leads to a slight increase in $C_{d}$ (less than $10 \%$ for $R \leq 50 \mathrm{~nm}$, but up to $15 \%$ for $R=100 \mathrm{~nm}$ ). A very similar trend was revealed in an analytical solution for an elastic plate [45]. This fact could explain the slight underestimation of $C_{d}$ by the simulations (see Fig. 7), particularly for $100 \mathrm{~nm}$ (see Fig. 4). However, other effects could also alter the quite sensitive value of $C_{d}$. Experimentally, one can modify the lipids forming the liposome and also the temperature, making the liposomes less or more "rigid." However, modifying the liposome rigidity naturally implies a change in the twodimensional fluidity of the membrane, which, physically, is an independent mechanism of dissipation [46]. Previous work where liposomes were directly tethered to a neutravidin layer by means of biotinylated lipids showed that stiffening of the bilayer by addition of cholesterol results in a higher acoustic ratio [47]. Thus, although the question remains open, experiments also indicate that the acoustic effects of the rigidity and fluidity of the membrane are of second order. An important message from this work (see Figs. 5 and 8 ) is that increasing the acoustic ratio (or $C_{d}$ ) does not imply further amplification of the acoustic signal (i.e., of the complex impedance). As a matter of fact, close to the wall, the impedance is high but $C_{d}$ is small, while far from the wall, the frequency shift tends to zero and $C_{d}$ increases (and diverges at some zero-frequencycrossing "height" [45]). This explains the increase in $C_{d}$ for $R=100 \mathrm{~nm}$ in Fig. 7 as the frequency is increased, and the same effect affects the results for large $L_{\mathrm{DNA}}$ and $R$ (Fig. 4). To disentangle all this subtle information hidden beneath the complex QCM hydrodynamics requires a combined effort in terms of experiments, simulations, and hydrodynamic theory. This paper represents a step in this direction.

\section{ACKNOWLEDGMENTS}

We acknowledge the EU Commission for the FETOPEN CATCH-U-DNA project. R.D-B acknowledges support from spanish goverment grant MINECO via the project FIS2017-86007-C3-1-p.

\section{APPENDIX A: NUMERICAL MODEL}

\section{Fluid solver}

Our numerical model is based on a fluctuating hydrodynamic solver for compressible flow, equipped with the immersed boundary method to couple the dynamics of the fluid and the structure. A detailed explanation of the method can be found in Refs. [31,32,35]. The code is called FLUAM, is written in CUDA, and runs on GPU architectures; it is available at a GitHub repository [33]. The integration scheme is second-order accurate in space and time, and the spatial discretization is based on a staggered grid [34] of size $h$. Boundary conditions for the top and bottom walls are imposed using a ghost cell [35]. This enables us to easily impose a tangential flow velocity at the bottom wall. The tangential velocity gradient in the wall cells $\left(\partial v_{x} / \partial y\right)_{y=0}$ is calculated using second-order interpolation from the values of the fluid-cell velocity above the wall position $y=0$. At each sampling time, we average $\eta\left(\partial v_{x} / \partial y\right)_{y=0}$ over all the fluid cells at the surface to obtain the fluid traction (shear stress) at the "resonator" surface. This leads to the impedance, as explained in the main text.

\section{Correspondence of units}

To map the code units (denoted by a subscript $c$ ) to International System (SI) units, we start by selecting a reference length $\ell$. We choose $\ell=\delta / 12$, which equals $7.917 \mathrm{~nm}$ for a penetration length of $\delta=95 \mathrm{~nm}$, corresponding to $35 \mathrm{MHz}$. We note that the calculations presented in the main text are carried out with a grid size of $h=0.5 \ell$, corresponding to 24 fluid cells per $\delta$. The code units of mass $M$ and time $\tau$ match the density and kinematic viscosity of water at $T=25^{\circ} \mathrm{C}$. In particular, $M=\rho_{c} \ell^{3}$ follows from $\rho_{c} M / \ell^{3}=\rho_{\mathrm{SI}} \mathrm{kg} / \mathrm{m}^{3}$ with $\rho_{\mathrm{SI}} \approx 10^{3}$ (water), and we take $\rho_{c}=1$. Choosing a value for the kinematic viscosity $v_{c}$ in the code and matching the kinematic viscosity of water so that $v_{c} \ell^{2} / \tau=v_{\mathrm{SI}} \mathrm{m}^{2} / \mathrm{s}$ yields the time scale $\tau$. From these fundamental units (mass, length, and time), the code values for the QCM frequency $f_{c}$ and the thermal energy $\left(k_{B} T\right)_{c}$ can be easily obtained. Table I shows the magnitudes and the mapping of units used.

\section{Molecular structures}

\section{a. Liposomes}

Liposomes are modeled using an elastic-network model (see Fig. 2). The membrane is created by beginning with a close-packed arrangement of spheres connected to their nearest neighbors with harmonic springs. By changing the value of the spring constant $k_{L}$, we can tune the bending rigidity of the liposome membrane. In this paper, we consider $k_{L}=100 \mathrm{M} / \tau$, which corresponds to a quite rigid particle $\left(k_{L} \ell^{2}>10^{5} k_{B} T\right.$ for $\left.T=300 \mathrm{~K}\right)$. The size of the beads in the arrangement is fixed by the spatial resolution 
$h$ of the Eulerian fluid mesh. The calculations presented thereby correspond to $h=0.5 \ell=3.958 \mathrm{~nm}$. We use these connected spheres as building blocks for the membrane simply by selecting only the spheres contained between two concentric spheres with radii equal to the inner and outer radii of the liposome membrane. The number of beads required to build a liposome increases as $(R / h)^{2}$ and is about 6000 beads for a liposome of radius $R=50 \mathrm{~nm}$.

\section{b. DNA strand}

The DNA is modeled using the same type of beads as for the liposome. The model reproduces a semiflexible polymer with a bending rigidity

$$
k_{\mathrm{bend}}=k_{B} T\left(\ell_{P} / \sigma\right)
$$

and a persistence length $\ell_{P}=50 \mathrm{~nm}$, evaluated at $k_{B} T=$ $4 \times 10^{-21} \mathrm{~J}$ (the thermal energy at room temperature). It consists of a series of harmonic springs between consecutive beads (at a distance $r=\left|\mathbf{R}_{i+1}-\mathbf{R}_{i}\right|$ ) with an equilibrium distance $r_{0}=\sigma$, i.e.,

$$
U_{\mathrm{sp}}(r)=\frac{k_{1}}{2}\left(r-r_{0}\right)^{2},
$$

and a three-body potential based on the angle $\theta$ between two consecutive bonds,

$$
U_{\text {angle }}(r)=-\frac{k_{\text {bend }}}{2} \theta^{2},
$$

where we use $k_{1}=100$ to represent an almost unstretchable chain. We impose an excluded-volume interaction between the DNA and the liposome beads via the Weeks-Chandler-Anderson (WCA) potential (with excluded diameter $\sigma=2 h$ ). The DNA chain is connected to the liposome via a harmonic spring, and so it is free to rotate. In practice, the range of angles formed between the DNA and the liposome tangent plane is slightly reduced due to the steric repulsion between beads (to about $\left.\left[-70^{\circ}, 70^{\circ}\right]\right)$. The other end of the DNA chain is linked to the QCM surface via a harmonic potential so that the chain is free to rotate at the linker point (corresponding to zero tilt energy). We check that the tilt energy [43] at the linker-DNA connection slightly alters the liposome height distribution $P(y)$ but does not significantly affect the acoustic ratio.

\section{Monte Carlo sampling of initial configurations and height probability density $P(y)$}

A pool of equilibrated initial configurations of individual liposome-DNA complexes is extracted from MC sampling at $T=300 \mathrm{~K}$. In these MC simulations, the liposome model is simplified to a single bead of radius $R$. The excluded-volume interaction between the DNA beads and the single-bead liposome is modeled using a WCA potential (i.e., a purely repulsive truncated Lennard-Jones potential) modified to be a function of the distance between the DNA bead and the liposome surface, $r-\sigma_{\text {eff }}$ with $\sigma_{\text {eff }}=\sigma / 2+R$, where $r$ is the center-to-center distance, i.e.,

$$
U_{\mathrm{EV}}(r)=4 \epsilon\left[\left(\frac{r}{r_{i 0}-\sigma_{\mathrm{eff}}}\right)^{12}-\left(\frac{r}{r_{i 0}-\sigma_{\mathrm{eff}}}\right)^{6}\right],
$$

with $\epsilon=1$.

To accelerate the $\mathrm{MC}$ sampling, we proceed as follows. (i) The DNA bead model is first generated by consecutively placing beads of radius $h$ at similar relative distances along a random walk with persistence $50 \mathrm{~nm}$. The beads are placed such that their separation is given by the equilibrium distance $\left(r_{0}\right)$ of the model [see Eq. (A1)]. Details of this procedure are explained in Ref. [48]. This step is rejected if a bead crosses the wall (if $y_{i}<0$ ) or if different beads in the chain overlap. (ii) The liposome, constructed from a single bead of radius $R$, is then placed at a distance $R+h$ from the last DNA bead. This step is rejected if the liposome crosses the wall $(y<R)$ or if a DNA bead crosses the outer liposome boundary (the distance between any DNA bead and the liposome center should be larger than $R+h$ ). The liposome-wall interaction is modeled as a purely steric repulsion $[P(y)=0$ for $y<R]$. This choice is motivated by several observations: First, electrostatic interactions with the wall are expected to be small because of the small charge of the neutravidin layer and the zero charge of POPC lipids (their zwitterionic head group is, moreover, strongly screened by the PBS saline buffer). Second, dispersion forces leading to physisorption are ruled out, upon comparison with experiments done with nontethered liposomes. Finally, theory and simulations also confirm that the acoustic ratio of adsorbed particles shows radically different trends when compared with that of suspended particles (see Fig. 8).

\section{APPENDIX B: CONVERGENCE}

\section{Stokes flow}

The impedance of the base fluid is needed to extract the analyte impedance $\left(\mathcal{Z}_{L+\mathrm{DNA}}=\mathcal{Z}-\mathcal{Z}_{0}-\mathcal{Z}_{\mathrm{DNA}}\right)$. A first check of the accuracy of the code is to compare the numerical and analytical values of the impedance of a Newtonian fluid, the latter given by the Gordon-Kanazawa result [17] $\mathcal{Z}_{0}=\alpha \eta$ with $\alpha=(i-1) / \delta$. We use tall boxes (with a length in the vertical direction $L_{y} \approx 5.3 \delta$ ), for which the finite-size correction to the fluid impedance is very small. As shown in Fig. 9, the relative deviation $\left(\mathcal{Z}_{f}^{(\text {num })}-\mathcal{Z}_{0}\right) / \mathcal{Z}_{0}$ between the analytic and numerical fluid impedances is less than or about $10^{-3}$ for $h=0.5 \ell$ (corresponding to $h / \delta=1 / 24$ in the figure). 

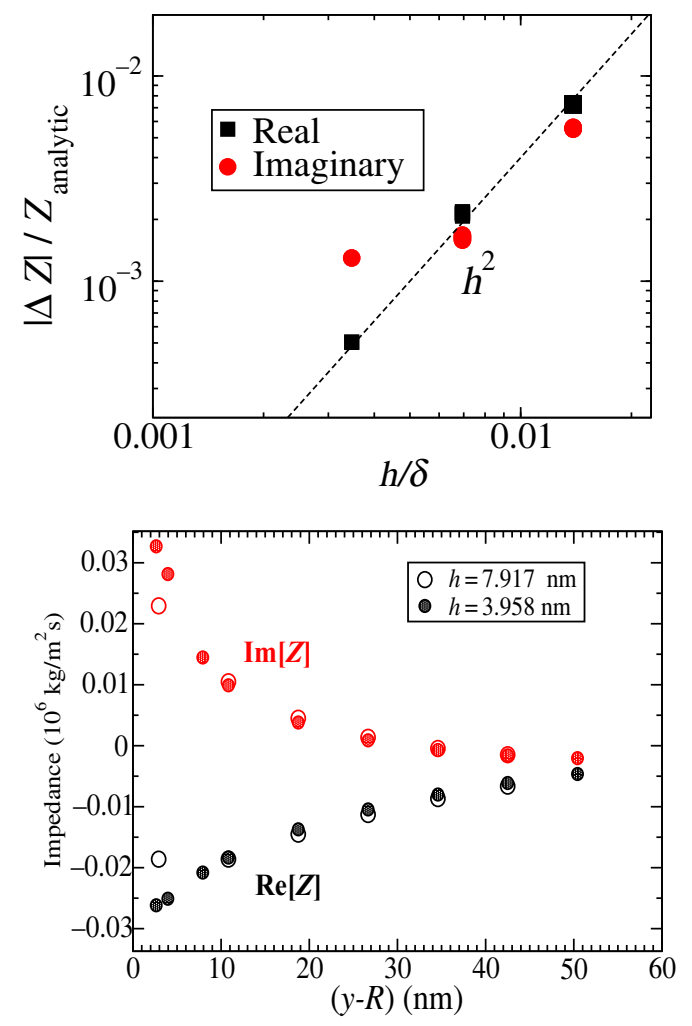

FIG. 9. (Top) Relative difference between the analytical value $Z_{\text {fluid }}=(i-1) \eta / \delta$ and the numerical value of the impedance of a Newtonian fluid versus the scaled mesh size $h / \delta$. (Bottom) Impedance of a freely suspended liposome of radius $R=$ $50 \mathrm{~nm}$ obtained for two mesh resolutions $h$ as a function of the liposome-wall (surface-to-surface) distance $y-R$.

\section{Liposome impedance}

Concerning the convergence of the liposome impedance, Fig. 9 (bottom panel) illustrates a study of this quantity versus the liposome-wall distance $y-R$, which compares results for $h=7.917 \mathrm{~nm}$ and $h=3.958 \mathrm{~nm}$. The greater precision of $h=0.5 \ell$ increases the resolution of the flow close to the wall, up to distances of about $4 \mathrm{~nm}$.

\section{APPENDIX C: RESULTS FOR DNA}

\section{Unloaded DNA tethers}

To follow the experimental procedure, we extract the contribution of the unloaded DNA strand $\mathcal{Z}_{\mathrm{DNA}}$ from the total impedance to obtain the sought liposome-DNA impedance, i.e., $\mathcal{Z}_{L+\mathrm{DNA}}=\mathcal{Z}-\mathcal{Z}_{0}-\mathcal{Z}_{\mathrm{DNA}}$. As in the experiments, the impedance of the dsDNA strands is much smaller than that of the liposomes considered, and single (unloaded) DNA strands (at the same coverage as the liposomes) will not be detected at the resolution of the code. Therefore we place a number $N_{\text {DNA }}$ of DNA strands, equilibrated according to the procedure described in Sec. A 4, on a smaller surface area $L^{2}$ (keeping $L_{y}=$ $5.3 \delta$ ). In agreement with experiments [21], the scaled

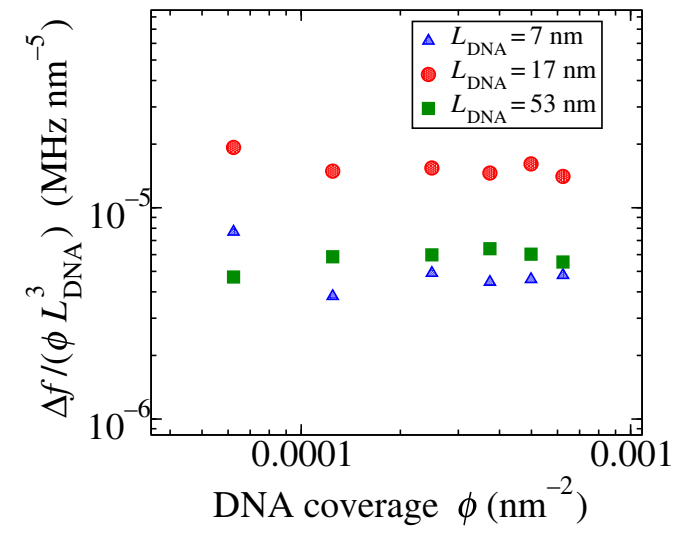

FIG. 10. Frequency shift created by DNA tethers divided by the DNA coverage $\phi=N_{\mathrm{DNA}} / L^{2}$, scaled by $L_{\mathrm{DNA}}^{3}$, plotted against the coverage $\phi$. The dissipation is also almost independent of the coverage. The values of the acoustic ratio $-\Delta D / \Delta f$ (in units of $10^{6} / \mathrm{Hz}$ ) are $0.0185 \pm 0.005$ and $0.009 \pm 0.001$ for $L_{\mathrm{DNA}}=53$ and $17 \mathrm{~nm}$, respectively, and are about half the experimental values $(0.03$ and 0.018$)$.

impedance $\mathcal{Z}_{\mathrm{DNA}} / \phi$ is observed to be independent of the surface coverage, as shown in Fig. 10. Also in agreement with experiments, the DNA acoustic ratio is observed to increase with the DNA length as $C_{d \mathrm{DNA}} \sim L_{\mathrm{DNA}}^{\alpha}$, with $\alpha \approx 3$, which is expected for $L_{\mathrm{DNA}}<150 \mathrm{~nm}$ [21]. However, the simulated values of $C_{d \text { DNA }}$ are about half the experimental values. To extract the DNA impedance from the liposome-DNA impedance, we note that the present analysis for DNA requires using the same resolution as for the $L$-DNA complex. Quite probably, these differences arise from the limited spatial resolution that we use here to model the DNA (with a bead diameter of about $h \approx 4 \mathrm{~nm}$ ). A numerical study focusing on the QCM response of the DNA alone would certainly require smaller values of $h$ (finer resolution), and this is left for future work.

\section{Impedance of anchored and free liposomes}

The ansatz in Eq. (2) for the impedance of a free liposome as a function of the liposome height can be written as

$$
\mathcal{Z}_{L}(y)=Z_{S}\left[(A+i B) \exp [-2 \alpha y]+\frac{(i-1) C \delta}{(y-R)}\right],
$$

where the stresslet impedance is defined as $Z_{S}=$ $(20 \pi / 3) R^{3} \eta /\left(\delta^{2} L^{2}\right)$. Figure 11 shows the best fits for $A$ and $B$ (real numbers) and for $(i-1) C$ (where $C$ is complex; see the main text), obtained from fits to the real and imaginary parts of the impedance for different sphere radii and forcing frequencies in the range $17.5-140 \mathrm{MHz}$. The values of $A$ and $B$ obtained from $\operatorname{Re}[Z]$ and $\operatorname{Im}[Z]$ are quite consistent with each other and can be fitted using functions of $(R / \delta)^{2}$. The parameter $C$ determines the strength of the near flow field, which has different forms for the real and 

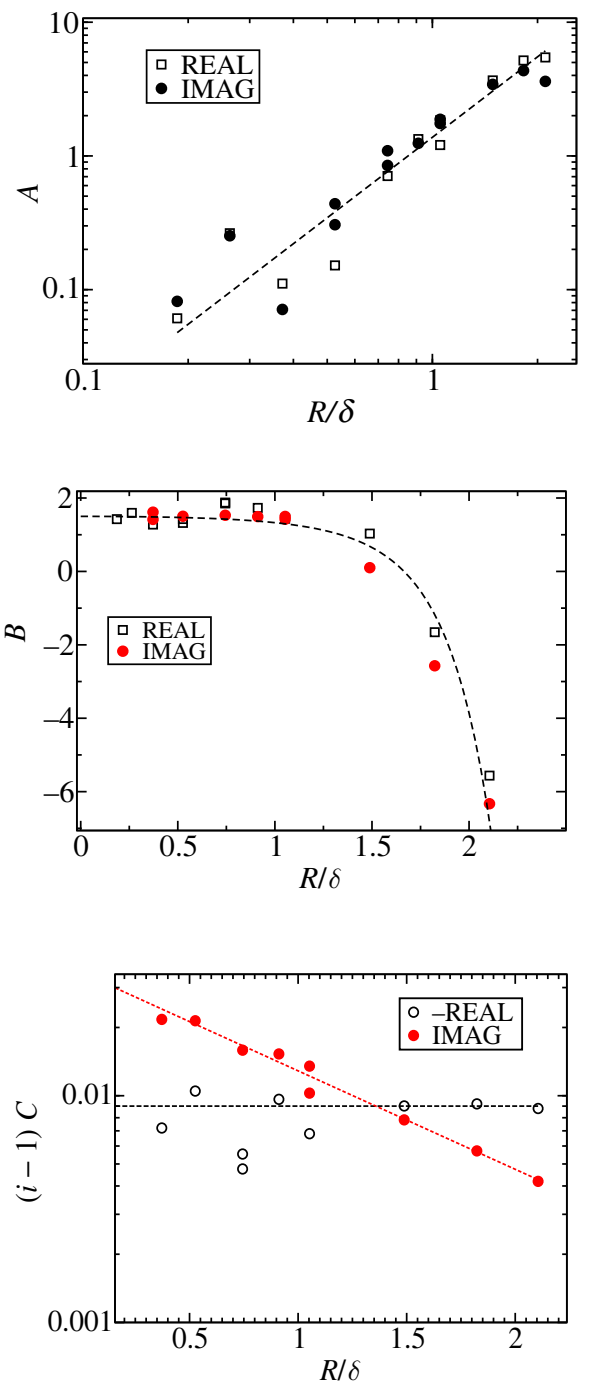

FIG. 11. Values of the parameters in Eq. (C1) obtained from best fits to simulation results for the impedance of free spheres, for different sphere radii and forcing frequencies. The results from fits to $\operatorname{Re}[Z]$ and $\operatorname{Im}[Z]$ are denoted by "REAL" and "IMAG." The dashed lines correspond to (top) $A=1.4(R / \delta)^{2}$, (middle) $B=1.6-0.1 \exp \left[(R / \delta)^{2}\right]$, and (bottom) $\operatorname{Re}[(1-i) C]=0.009, \operatorname{Im}[(i-1) C]=0.035 \exp [-R / \delta]$.

imaginary parts of the impedance. Note that we use a fixed value of $h$, so that the number of beads (of size $h$ ) used to resolve the liposome surface increases as $R^{2}$. In this sense, $R=100 \mathrm{~nm}$ corresponds to the best liposome resolution.

\section{Contribution of the DNA to the liposome-DNA impedance}

The contribution of the DNA to the liposome-DNA impedance is estimated as $\mathcal{Z}_{L+\mathrm{DNA}}^{\text {(DNA) }}=\mathcal{Z}_{L+\mathrm{DNA}}-\mathcal{Z}_{L}$, where $\mathcal{Z}_{L}$ is the impedance of a free liposome (without an anchor). The average of $\mathcal{Z}_{L+\mathrm{DNA}}^{\text {(DNA) }}$ for different values of $y$ (the liposome height) is plotted in Fig. 12 (the

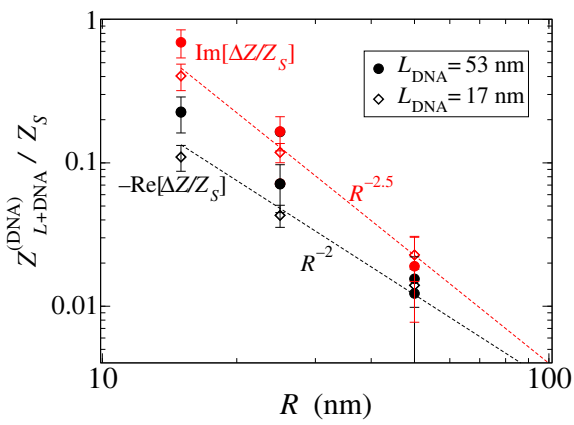

FIG. 12. Difference between the impedance of a liposomeDNA complex and that of a free liposome, $\mathcal{Z}_{L+\mathrm{DNA}}^{\text {(DNA) }} \equiv$ $\left(\mathcal{Z}_{L+\mathrm{DNA}}-\mathcal{Z}_{L}\right)$, scaled by $Z_{S}=(20 \pi / 3) \eta R^{3} /\left(L^{2} \delta^{2}\right)$ (see text). The error bars indicate one third of the standard deviation of $\mathcal{Z}_{L+\mathrm{DNA}}^{\text {(DNA) }}$ for 20 configurations.

error bars correspond to one third of the standard deviation for 20 configurations). The results scale roughly as $\mathcal{Z}_{L+\mathrm{DNA}}^{\mathrm{(DNA})} \approx\left(-7.2 R^{-2}+i 83 R^{-2.5}\right) L_{\mathrm{DNA}}^{0.5} Z_{S}$ (with $R$ and $L_{\mathrm{DNA}}$ in nanometers). As $Z_{S} \propto R^{3}$, this indicates that $\operatorname{Re}\left[\mathcal{Z}_{L+\mathrm{DNA}}^{(\mathrm{DNA})}\right] \sim R$, while $\operatorname{Im}\left[\mathcal{Z}_{L+\mathrm{DNA}}^{(\mathrm{DNA})}\right]$ increases as $R^{0.5}$.

\section{Effect of the liposome coverage (lateral box side)}

Figure 13 shows $\Delta f$ and the dissipation factor $\Delta D$ plotted against the liposome surface coverage $\phi=\pi(R / L)^{2}$. Results for two spatial resolutions $h$ are included for liposomes of radius $R=50 \mathrm{~nm}$ to illustrate the numerical convergence. Interestingly, $\Delta f$ and $\Delta D$ increase almost linearly with $\phi$. This fact could be a consequence of the hydrodynamics of neutrally buoyant particles; however, this observation should be analyzed in detail using theoretical hydrodynamics. Yet, the simulations reveal a small but noticeable deviation from linearity, which leads to a decrease in the acoustic ratio $\Delta D / \Delta f$ with $\Delta f$, similarly

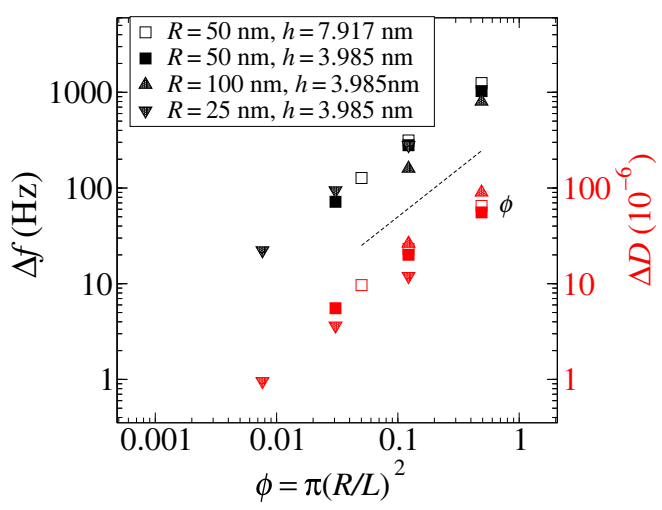

FIG. 13. $\Delta f$ and $\Delta D$ (dissipation factor) plotted against the surface coverage $\phi=\pi(R / L)^{2}$ corresponding to simulations for $L_{\mathrm{DNA}}=17 \mathrm{~nm}$ and different liposome radii and spatial resolutions $h$. 
to that observed in experiments [2] (see Fig. 3). Unfortunately, we do not have an independent experimental measurement to complement the calculations other than this indication of the frequency change $\Delta f$.

[1] J. Krim, Friction and energy dissipation mechanisms in adsorbed molecules and molecularly thin films, J. Adv. Phys. 61, 155 (2012).

[2] Diethlm Johannsmann, The Quartz Crystal Microbalance in Soft Matter Research, Fundamentals and Modeling (Springer, London, 2015).

[3] N. L. Bragazzi, D. Amicizia, D. Panatto, D. Tramalloni, I. Valle, and R. Gasparini, in Advances in Protein Chemistry and Structural Biology (Elsevier, Amstermdam, 2015), Vol. 101, p. 149.

[4] M. Rodahl, F. Hook, C. Fredriksson, C. A. Keller, A. Krozer, P. Brzezinski, M. Voinova, and B. Kasemo, Simultaneous frequency and dissipation factor QCM measurements of biomolecular adsorption and cell adhesion, Faraday Discuss. 107, 229 (1997).

[5] Ilya Reviakine, Diethelm Johannsmann, and Ralf P. Richter, Hearing what you cannot see and visualizing what you hear: Interpreting quartz crystal microbalance data from solvated interfaces, Anal. Chem. 83, 8838 (2011).

[6] C. A. Keller and B. Kasemo, Surface specific kinetics of lipid vesicle adsorption measured with a quartz crystal microbalance, Biophys. J. 75, 1397 (1998).

[7] Achilleas Tsortos, George Papadakis, and Electra Gizeli, On the hydrodynamic nature of DNA acoustic sensing, Anal. Chem. 88, 6472 (2016).

[8] M. Saitakis and E. Gizeli, Acoustic sensors as a biophysical tool for probing cell attachment and cell/surface interactions, Cell. Mol. Life Sci. 69, 357 (2012).

[9] Edurne Tellechea, Diethelm Johannsmann, Nicole F. Steinmetz, Ralf P. Richter, and Ilya Reviakine, Modelindependent analysis of QCM data on colloidal particle adsorption, Langmuir 25, 5177 (2009).

[10] Tao Liu, Ji'an Tang, and Long Jiang, The enhancement effect of gold nanoparticles as a surface modifier on DNA sensor sensitivity, Biochem. Biophys. Res. Commun. 313, 3 (2004).

[11] L. B. Nie, Y. Yang, S. Li, and N. Y. He, Enhanced DNA detection based on the amplification of gold nanoparticles using quartz crystal microbalance, Nanotechnology $\mathbf{1 8}$, 305501 (2007).

[12] Dujuan Li, Jianping Wang, Ronghui Wang, Yanbin Li, Daad Abi-Ghanem, Luc Berghman, Billy Hargis, and Huaguang Lu, A nanobeads amplified QCM immunosensor for the detection of avian influenza virus H5N1, Biosens. Bioelectron. 26, 4146 (2011).

[13] Yulong Jin, Yanyan Huang, Guoquan Liu, and Rui Zhao, Gold nanoparticle-sensitized quartz crystal microbalance sensor for rapid and highly selective determination of $\mathrm{Cu}$ (II) ions, Analyst 138, 5479 (2013).

[14] Ye Yang, Guillaume Poss, Yini Weng, Runzhang Qi, Hanrui Zheng, Nikolaos Nianias, Euan R. Kay, and Stefan Guldin, Probing the interaction of nanoparticles with small molecules in real time via quartz crystal microbalance monitoring, Nanoscale 11, 11107 (2019).
[15] F. Patolsky, K. T. Ranjit, A. Lichtenstein, and I. Willner, Dendritic amplification of DNA analysis by oligonucleotide-functionalized Au-nanoparticles, Chem. Commun. 12, 1025 (2000).

[16] X. C. Zhou, S. J. O'Shea, and S. F. Y. Li, Amplified microgravimetric gene sensor using $\mathrm{Au}$ nanoparticle modified oligonucleotides, Chem. Commun. 11, 953 (2000).

[17] K. Keiji Kanazawa and Joseph G. Gordon, Frequency of a quartz microbalance in contact with liquid, Anal. Chem. 57, 1770 (1985).

[18] A. J. Ricco and S. Martin, Acoustic wave viscosity sensor, J. Appl. Phys. Lett. 50, 1474 (1987).

[19] D. Johannsmann, K. Mathauer, G. Wegner, and W. Knoll, Viscoelastic properties of thin films probed with a quartzcrystal resonator, Phys. Rev. B 46, 7808 (1992).

[20] M. V. Voinova, M. Rodahl, M. Jonson, and B. Kasemo, Viscoelastic acoustic response of layered polymer films at fluid-solid interfaces: Continuum mechanics approach, Phys. Scripta 59, 391 (1999).

[21] Achilleas Tsortos, George Papadakis, Konstantinos Mitsakakis, Kathryn A. Melzak, and Electra Gizeli, Quantitative determination of size and shape of surface-bound DNA using an acoustic wave sensor, Biophys. J. 94, 2706 (2008).

[22] V. Raptis, A. Tsortos, and E. Gizeli, Theoretical Aspects of a Discrete-Binding Approach in Quartz-Crystal Microbalance Acoustic Biosensing, Phys. Rev. Appl. 11, 034031 (2019).

[23] G. Papadakis, A. Tsortos, and E. Gizeli, Acoustic characterization of nanoswitch structures: Application to the DNA holliday junction, Nano Lett. 10, 5093 (2010).

[24] Pablo Mateos-Gil, Achilleas Tsortos, Marisela Vélez, and Electra Gizeli, Monitoring structural changes in intrinsically disordered proteins using QCM-D: Application to the bacterial cell division protein ZipA, Chem. Commun. 52, 6541 (2016).

[25] R. Akter, C. K. Rhee, and M. A. Rahman, A highly sensitive quartz crystal microbalance immunosensor based on magnetic bead-supported bienzymes catalyzed mass enhancement strategy, Biosens. Bioelectron. 66, 539 (2015).

[26] Jan-W. Thies, Bettina Thürmann, Anke Vierheller, and Andreas Dietzel, Particle-based microfluidic quartz crystal microbalance (QCM) biosensing utilizing mass amplification and magnetic bead convection, Micromachines 9, 194 (2018).

[27] Dimitra Milioni, Pablo Mateos-Gil, George Papadakis, Achilleas Tsortos, Olga Sarlidou, and Electra Gizeli, An acoustic methodology for selecting highly dissipative probes for ultra-sensitive DNA detection, Anal. Chem. 92, 8186 (2020).

[28] D. Johannsmann and G. Brenner, Frequency shifts of a quartz crystal microbalance calculated with the frequencydomain lattice-Boltzmann method: Application to coupled liquid mass, Anal. Chem. 87, 7476 (2015).

[29] Jurriaan J. J. Gillissen, Joshua A. Jackman, Seyed R. Tabaei, and Nam-Joon Cho, A numerical study on the effect of particle surface coverage on the quartz crystal microbalance response, Anal. Chem. 90, 2238 (2018).

[30] M. Rodahl, F. Hook, A. Krozer, P. Brzezinski, and B. Kasemo, Quartz crystal microbalance setup for frequency and Q-factor measurements in gaseous and liquid environments, Rev. Sci. Instrum. 66, 3924 (1995). 
[31] F. Balboa Usabiaga, Ignacio Pagonabarraga, and Rafael Delgado-Buscalioni, Inertial coupling for point particle fluctuating hydrodynamics, J. Comput. Phys. 235, 701 (2013).

[32] Florencio Balboa Usabiaga, Rafael Delgado-Buscalioni, Boyce E. Griffith, and Aleksandar Donev, Inertial coupling method for particles in an incompressible fluctuating fluid, Comput. Methods Appl. Mech. Eng. 269, 139 (2014).

[33] F. Balboa-Usabiaga, FLUAM https://github.com/fbusa biaga/fluam/.

[34] Florencio Balboa, John B. Bell, Rafael Delgado-Buscalioni, Aleksandar Donev, Thomas G. Fai, Boyce E. Griffith, and Charles S. Peskin, Staggered schemes for fluctuating hydrodynamics, Multiscale Model. Simul. 10, 1369 (2012).

[35] Florencio Balboa Usabiaga, Ph.D. thesis, Universidad Autonoma de Madrid, 2014.

[36] P. Mazur and D. Bedeaux, A generalization of Faxén's theorem to nonsteady motion of a sphere through an incompressible fluid in arbitrary flow, Physica 76, 235 (1974).

[37] Dimitra Milioni, Achilleas Tsortos, Marisela Velez, and Electra Gizeli, Extracting the shape and size of biomolecules attached to a surface as suspended discrete nanoparticles, Anal. Chem. 89, 4198 (2017).

[38] A. Simha, J. Mo, and P. J. Morrison, Unsteady stokes flow near boundaries: The point-particle approximation and the method of reflections, J. Fluid Mach. 841, 883 (2018).

[39] S. Kim and S. J. Karrila, Microhydrodynamics: Principles and Selected Applications (Dover Pulications Inc. Mineola, New York, 2005).
[40] C. Pozrikidis, Fluid Dynamics: Theory, Computation, and Numerical Simulation (Oxford University Press, New York, 2011).

[41] B. U. Felderhof, Hydrodynamic force on a particle oscillating in a viscous fluid near a wall with dynamic partial-slip boundary condition, Phys. Rev. E 85, 046303 (2012).

[42] We have verified that the divergence in Eq. (2) saturates at contact, as expected.

[43] Ka-Yiu Wong and Montgomery B. Pettitt, Orientation of DNA on a surface from simulation, Biopolymers 73, 570 (2004).

[44] Ling Huang, Erkin Seker, James P. Landers, Matthew R. Begley, and Marcel Utz, Model-independent analysis of QCM data on colloidal particle adsorption, Langmuir 26, 11574 (2010).

[45] M. Melendez, A. Vazquez-Quesada, and R. DelgadoBuscalioni, Load impedance of immersed layers on the quartz crystal microbalance: a comparison with colloidal suspensions of spheres (to be published).

[46] Sergio Panzuela and Rafael Delgado-Buscalioni, Solvent Hydrodynamics Enhances the Collective Diffusion of Membrane Lipids, Phys. Rev. Lett. 121, 048101 (2018).

[47] Kathryn A. Melzak, Florian Bender, Achilleas Tsortos, and Electra Gizeli, Probing mechanical properties of liposomes using acoustic sensors, Langmuir 24, 9172 (2008).

[48] Nerea Alcázar-Cano and Rafael Delgado-Buscalioni, A general phenomenological relation for the subdiffusive exponent of anomalous diffusion in disordered media, Soft Matter 14, 9937 (2018). 